\title{
EVOLUCION DEL SISTEMA DE ARRENDAMIENTO DE UN MONOPOLIO COMERCIAL: LAS CARNICERÍAS DE CRISTIANOS DE CÓRDOBA (SIGLOS XIII AL XV)
}

\author{
Jesús Padilla González \\ Universidad de Córdoba
}

Si en la Baja Edad Media cordobesa hay un conflicto socioeconómico significativo es, sin duda alguna, el de las "carnicerías de cristianos"; no sólo por su duración —que llega a superar el siglo XV y prolongarse hasta bien entrada la Era Contemporánea-, sino por sus implicaciones sociales, económicas, jurídicas, políticas, religiosas, institucionales, urbanísticas, etc., que al concurrir en él le dotan de una enorme complejidad y de una personalidad propia; por lo que, en gran parte, su historia es la Historia de sectores vitales de la sociedad cordobesa (1).

Mas si esta área de la vida urbana fue conflictiva, se debió, sustancialmente, al hecho de ser -en opinión del cabildo del concejo de la ciudad- una excepción de la norma: las carnicerias de cristianos cordobesas fueron monopolio del obispo y cabildo de la iglesia catedral -concedido en circunstancias un tanto ambiguas (2)-, cuando por derecho común deberian haber pertenecido al concejo de la ciudad (3). Este hecho fue la causa de que fuera dura y largamente contestado, tanto por los carniceros como por los propios capitulares del concejo (4).

Dejando aparte los muy diversos tipos de consideraciones que sobre las carnicerias podriamos hacer, en este breve artículo nos vamos a centrar en el análisis de uno de los aspectos claves para la comprensión de los conflictos que en torno a ellas surgieron $y$, por consiguiente, de la dinámica socio-económica de la Baja Edad Media cordobesa. Nos estamos refiriendo al sistema de arrendamiento de éstas y a su evolución a lo largo de los siglos XIII al XV. 
Como muy bien pone de manifiesto María Concepción Quintanilla Raso, "en las ciudades hispanomusulmanas, las rentas del comercio urbano en buena medida se hallaban en manos del monarca, y esta situación se mantuvo durante los primeros momentos de la dominación cristiana. Pero muy pronto, en el mismo siglo XIII, la monarquia comenzó a enajenar estas rentas para cederlas como mercedes a los particulares" (5).

Este será el caso que nos ocupa. Tras la conquista de Córdoba llevada a cabo por Fernando III en 1236, y el reparto subsiguiente del botín de guerra, el monarca retuvo para sí, entre otros bienes, la propiedad y, por tanto, las rentas de las dos carnicerías de la ciudad, las cuales se hallaban ubicadas: una, en la collación de Santa Maria, junto a la Alcaicería, cerca de la catedral; la otra, en la plaza de San Salvador, en la collación de San Andrés, ambas formando parte de dos de los núcleos comerciales más importantes de Córdoba.

En este contexto, el primer sistema de arrendamiento establecido para arrendar las tablas de las carnicerías consistió en ponerlas en almoneda anualmente junto con el amojarifazgo, haciéndose con ellas, como es obvio, aquel que más ofrecía (6). Asi se estuvo haciendo hasta que Alfonso $X$, en la segunda mitad de 1280 , las otorgó a los carniceros de la ciudad a cambio del pago de un tributo anual de $3,5 \mathrm{mrs}$. alfonsíes (7) y con las mismas condiciones que las habia concedido a los carniceros de SeviIla (8).

Más aún, no había transcurrido un año cuando, el 25 de julio de 1281, el mismo monarca las volvió a conceder al obispo y cabildo de la iglesia catedral -reservando para la Corona el derecho de alcabala-, junto con el diezmo que poseía en las Ollerías y Tinajerías de la ciudad, asi como las diez tiendas en las que se vendían las ollas -en definitiva, el monopolio de la comercialización de la carne y alfarería de Córdoba (9)—: Todo ello a cambio de unas tiendas propiedad de aquellos que, personalmente, el rey había ordenado derribar porque encontrándose adosadas a los mu ros de la iglesia de Santa María - es decir, a los muros de la gran mezquita catedral- degradaban considerablemente la majestuosidad y belleza del edificio (10). Noble gesto del refinado espíritu de un sabio rey, que acarrearía serios problemas para la ciudad.

A partir de esta donación se abrirá un largo período de enfrentamientos entre la Iglesia y el gremio de los carniceros: el obispo y cabildo catedralicio se empeñaron en que las tablas de las carnicerías debian ser puestas anualmente en almoneda. Los carniceros, oponiéndose a dicha pretensión, defendieron el parecer de que la Iglesia sólo debía cobrar el tributo de los tres mrs. y medio que para sí se había reservado el monarca.

La opinión del obispo y canónigos fue la que se impuso, a pesar de las protestas y denodados esfuerzos - no exentos muchas veces de violencia, coacciones y otros métodos heterodoxos tales como la huelga general, luchas callejeras, falsificaciones de documentos, etc.-, realizados 
tanto por los carniceros como por el pueblo y el concejo de la ciudad, pues a causa del procedimiento de arrendamiento de las carnicerias mencionado, sus tablas se vieron sometidas a una vertiginosa alza de sus precios al pasar de 3,5 mrs. a 14; de 14 a 500; de 500 a $600 \mathrm{mrs}$., y, finalmente, de 600 a 1.000 en el breve espacio de tiempo de 30 años -entre 1281 y 1311 - a consecuencia de lo cual se produjo en la ciudad un gran encarecimiento de la carne, asi como fuertes tensiones en su población (11).

No obstante, el sistema de arrendamiento propugnado por los clérigos terminó estableciéndose de manera incuestionable en la sentencia definitiva que el 25 de febrero de 1311 Fernando IV libró a favor de la Iglesia y en contra de los carniceros, y en la que se anulaba el privilegio que a éstos le fuera otorgado con anterioridad que a la Iglesia por Alfonso x (12).

Por una real provisión de Alfonso XI de 1331, en la que se pone de manifiesto la denuncia presentada por el deán y cabildo de la iglesia de Córdoba de que los carniceros se negaban a acudir a arrendar las tablas de las carnicerias e intentaban abrirlas a sus propias expensas, sabemos que a comienzos de la década de los treinta seguía vigiente el sistema de arrendamiento tradicional (13).

Mas esta modalidad no iba a durar mucho tiempo más, pues la coyuntura del siglo XIV no iba a ser muy propicia a partir de entonces; y no lo va a ser no por la presión ejercida sobre los detentadores de la exclusiva por los carniceros, ni tan siquiera por la del concejo que a partir de $1311 \mathrm{se}$ agudizó, sino por la coacción de las catastróficas circunstancias demográticas y económico-sociales del siglo.

Poseemos una muy deteriorada carta de arrendamiento --que estimamos fue realizada entre el 1336 y 1346 - por la que el obispo don Juan [Pérez], deán y cabildo arrendaron «por seis años» una tabla con su tienda, en la carnicería de San Salvador a Esteban Martínez por $310 \mathrm{mrs}$. anuales (14). Este documento es por nosotros considerado como el eslabón perdido en la evolución del sistema de arrendamiento imperante en el siglo XIII y primeras décadas del XIV y el que encontraremos a partir de la segunda mitad del siglo XIV, tan completamente diferente: el primero, el arrendamiento anual y en almoneda; el segundo, el procedimiento del alquiler «de por vida».

Las motivaciones que impulsarían a los clérigos a operar este sustancial cambio pudieran ser debidas a un intento de conformar a los carniceros después de la revuelta que se produjo en 1331, que se menciona en la provisión de Alfonso XI, lo que dudamos. Más nos inclinamos a pensar que vino forzada por el hecho de que la década de los treinta y de los cuarenta fue un período de gran inestabilidad económica en la ciudad, con fuertes crisis en los años 1336, 1342 y 1345 , sin olvidar el gran trauma colectivo de 1349, la epidemia de la Peste Negra (15), lo que, sin duda alguna, obligaría al cabildo catedralicio a ser más flexible en sus exigen- 
cias económicas, no sólo bajando el coste de los arrendamientos, sino haciéndolos más estables.

Documentalmente, no será hasta 1354 cuando por primera vez encontramos el testimonio escrito del que constituirá el sistema de arrendamiento definitivo para toda la Baja Edad Media cordobesa, aunque esto no quiera decir que, con posterioridad, no sufra algunas modificaciones no sustanciales. El 31 de octubre del referido año, el cabildo extendió una carta de arrendamiento "de por vida» a favor de Pedro Fernández, la primera tabla a mano derecha de las Carnicerias de San Salvador, por 813 mrs. de renta anual, tabla que había quedado vacante por muerte de Juan Abril, "que la tuuo de por uida». Razón esta que claramente nos indica que el sistema de arrendamiento mencionado era anterior a 1354, aunque no podamos precisar con certeza desde cuándo (16). A partir de esta fecha este procedimiento será el usual.

Si pensamos que el primer documento estuviese fechado en 1336, año primero del pontificado del obispo don Juan Pérez, y el segundo en 1354 , tendríamos que la fecha de la implantación del nuevo sistema de arrendamiento en las carnicerías se debió producir en el espacio máximo comprendido en este intervalo de 18 años; mas debemos tener en cuenta que ya antes de 1354 se había realizado, al menos, un arrendamiento de por vida.

Este hecho refleja, a nuestro entender, un cambio profundo y de gran trascendencia en la historia de las carnicerias, operado - cuestión esta que hay que tener en cuenta- en un período que, al menos documentalmente, no hubo ningún grave conflicto entre los carniceros y el cabildo que pudiera hacernos pensar que fue debido a dicha presión la que obligó a los canónigos a un cambio de actitud en este sentido. Lo que sí nos parece más que suficiente fue la enorme influencia que pudo ejercer -y observamos la coincidencia con el anterior cambio de procedimiento- la gravísima crisis de 1349: la aparición de la Peste Negra en Córdoba, que venía a culminar un largo periodo de ciclos cortos de inflexiones económicas (17).

Los estragos de la epidemia, así como el deseo de los clérigos de atraer o retener a los carniceros en sus puestos, sí creemos que serían fuertes motivos para operar un cambio en este sentido, y las secuelas de la Peste, pensamos, una sólida razón para consagrar el nuevo procedimiento.

Aunque, como hemos afirmado, este sistema fue el usual, no obstante, con posterioridad se introdujeron algunas modificaciones, tales como el arrendamiento "por dos vidas». Así, en 1396 el cabildo contrató a Bartolomé Sánchez y a su hijo Diego Sánchez, de 12 años, la quinta tabla de la carnicería de Santa María, según se entraba por la Alcaicería, y una casilla junto a ella por $60 \mathrm{mrs}$. de la moneda vieja «por dos vidas» (18). A nuestro parecer, esta tabla no deberia ser muy buena dado el precio por la que se 
contrata. Sin embargo, tenemos que decir que esta modalidad de arrendamiento se hicieron siempre cuando se trataba de padre e hijo.

No nos debemos de extrañar que, a partir de la segunda mitad del siglo XIV, los carniceros perdieran la agresividad que habian mostrado en el siglo XIII; la causa de ello, damos por descontado, fue la estabilización de los precios de las tablas de las carnicerías que supuso la generalización del sistema de arrendamiento vitalicio. Los alquileres a «dos vidas» sería un procedimiento de asegurar la rentabilidad de algunas tablas que por su situación en las carnicerías, o por su estado, no serían económicamente apetecibles para los carniceros y, por consiguiente, el cabildo corria el riesgo de no poderlas arrendar.

Sin embargo, nos ronda en el pensamiento una teoría que aún no hemos podido comprobar documentalmente por su dificultad, y que podría explicar este cambio de actitud del cabildo catedralicio: Se trataría de constatar un cambio de mentalidad económica operada en esta Institución en la segunda mitad del siglo XIV, que la haría más conservadora y menos agresiva, económicamente hablando; lo que le inclinaría a los arrendamientos a largo plazo y no a los sobresaltos de los arrendamientos anuales y en almoneda.

Un segundo aspecto importante a tener en cuenta en el estudio del sistema de arrendamiento es el análisis del propio mecanismo de arrendamiento, cuestión ésta que vamos a abordar a continuación:

La contratación de las tablas de carnicería tenía, al menos desde el siglo XIV, una doble fase - como era habitual en todo tipo de arrendamientos de bienes del cabildo-: la primera era el «remate», consistente en poner en almoneda la mencionada propiedad entre los propios eclesiásticos capitulares en varios cabildos sucesivos, generalmente tres. El importe de esta puja era destinado a la Mesa capitular; la segunda era el propio arrendamiento al carnicero, no ya en ni por el cabildo, sino por aquel clérigo que había ganado el remate, el cual, en nombre del deán y cabildo, ponía la propiedad en pública subasta. Era el llamado «recaudo». Procuraba, por supuesto, tener algún margen de beneficio (19).

Con posterioridad, la costumbre modificó este procedimiento introduciendo una pequeña novedad: cuando una tabla -o cualquier otra propiedad del cabildo- quedaba vacante, el carnicero o los individuos interesados en hacerse con ella buscaban a un canónigo, que representando sus intereses actuaba como su procurador en los cabildos en los que se realizaban los remates. Por ello, cuando el clérigo se quedaba con la propiedad en arrendamiento se hacía innecesaria la realización del recaudo en pública almoneda, porque ya estaba de antemano concertada. En estos casos, el clérigo cobraría un cierto corretaje que nos es muy difícil de precisar al no recogerse en los documentos de arrendamiento (20).

Las tablas y tiendas arrendadas podían ser transferidas, particular- 
mente, cuando se trataban de pasar de padre a hijo (21). Para ello se precisaba tener el consentimiento del cabildo, lo que se conseguía a través de un capitular del mismo, probablemente por mediación del que hiciera el remate y recaudo de la referida tabla o tienda.

Si se concedia la autorización, previa subida, o no, del precio del arrendamiento, el cabildo nombraba entre sus miembros una comisión que recibía la renuncia del carnicero saliente y hacía el recaudo al entrante (22).

Sin embargo, este procedimiento fue prohibido por el propio cabildo el 2 de agosto de 1396, “dada la continua devaluación y depreciación de las propiedades del cabildo que se arriendan a legos por irse renunciando unos en otros el arrendamiento de las mismas, en adelante se prohibe tal costumbre para poderlas arrendar en más precio» (23).

Un carnicero podía arrendar más de una tabla al mismo tiempo (24), fenómeno que condujo al proceso especulativo del subarriendo, detectado a principios del siglo XV y que deducimos de una de las cláusulas de un arrendamiento en la que se expresa concretamente la tajante prohibición de realizar tal operación sin contar con licencia previa del cabildo (25).

Los pagos de los arrendamientos se hacian por tercios y al mayordomo del comunal, so pena de la pérdida de la tabla o tienda, la cual, sin previo juicio y sin necesidad de mandamiento de alcalde o juez, volvería al cabildo, que tomaría de los bienes de los carniceros o de los de sus fiadores el doble del importe de la renta establecida en el contrato si en el plazo de un año no pagaba.

Las tablas se arrendaban «a fuero de almojarifazgo», con las mismas condiciones, penas, usos y costumbres con que el rey y sus contadores mayores arrendaban el almojarifazgo y demás rentas que el monarca tenía en la ciudad; testimonio esto del origen y procedencia del monopolio detentado por el obispo y cabildo, fruto de la enajenación de rentas reales.

Los arrendatarios estaban obligados a conservar las tablas y tiendas en perfecto estado, corriendo a su cargo las reparaciones y mantenimiento de lo arrendado. Todos los años, los veedores del cabildo inspeccionaban las carnicerías. Si encontraban en mal estado algo, por mal uso o trato, daban al arrendatario un plazo fijo para que en dicho tiempo subsanasen los desperfectos observados, bajo amenaza de cancelación del arrendamiento, tomando en estos casos, el cabildo, de los bienes propios de aquél o de sus fiadores tanto cuanto importase la reparación. Por estas causas no se podía apelar a ningún tribunal ni juez, como se especifican en las cláusulas de las cartas de arrendamientos.

Al concluir un contrato bien por finalización del plazo temporal por el que se había firmado, bien por renuncia voluntaria, por impago o por muerte de los arrendatarios, los bienes arrendados debian ser devueltos 
al cabildo «adobados, bien reparados, en fiesta y a vista de maestros albannies y carpinteros", haciéndose de ello garante los propios bienes del arrendatario o los de sus fiadores (26).

El rigor en el límite cronológico que nos hemos impuesto en este trabajo - la Baja Edad Media - nos impide seguir adelante en la consideración evolutiva del sistema de arrendamiento de las carnicerias de Córdoba. Sistema que sufrió sustanciales modificaciones a partir del siglo XVI, pero cuya consideración, en estos momentos, estaría fuera de tono $y$, además, supondria desbordar el marco de nuestras pretensiones.

No obstante, creemos que con lo expuesto en este breve artículo hemos aportado algunos datos sustanciales para la comprensión del sistema económico imperante en la Córdoba de los siglos XIII al XV, pues muchos de los aspectos señalados podrian hacerse extensivos a otros sectores económicos - ollerías, tiendas de diverso género, hornos, mesones, etc.- del área de influencia del omnipresente poder económico detentado por el Cabildo de la catedral de Córdoba. 


\section{NOTAS}

(1) Sobre este tema hemos estudiado los siglos XIII, XIV y XV. Nos falta aún por investigar el XVI. Este trabajo lo hemos centrado en cinco puntos esenciales: El primero, una narración histórica de todos los acontecimientos acaecidos en torno a las Carnicerías; el segundo estudia el grupo social de los carniceros, sus condiciones económicas, profesionales, evolución, etc.; el tercero analiza la propia Institución, funcionamiento, ordenanzas, etc. En el cuarto investigamos aspectos económicos de la misma, y, finalmente, terminamos con las consideraciones toponimicas y urbanisticas de las carnicerias y las transformaciones que sufrieron a lo largo de los siglos que vemos.

(2) Alfonso $X$ concedió las carnicerías de cristianos de Córdoba al obispo y cabildo catedralicio el 25 de julio de 1281 (Archivo de la catedral de Córdoba -en adelante, ACC-, Cajón W, n. 1; NIETO CUMPLIDO, M., Corpus Mediaevale Cordubense, 1281, julio 25. Córdoba. Un amplio extracto de todos los documentos que citaremos en el presente artículo ha sido incluido en esta obra; deseamos agradecer desde estas páginas a don Manuel Nieto la amabilidad de habernos dejado consultar la parte inédita de este Corpus cuyos dos primeros tomos ya están en las librerias, Corpus Mediaevale Cordubense, I (1106-1255) (Cordoba, 1979) y $/ 1(1256,1277)$ (Córdoba, 1980). En adelante citaremos CMC, utilizando la fecha como signatura). Poco menos de un año antes se las había otorgado a los carniceros de la ciudad (Biblioteca de la Catedral de Córdoba -en adelante, BCC-, Ms. 125, ff. 15 r.-16 v.).

(3) Cfr. una real ejecutoria de 30 de junio de 1578, dada en la Real Chancillería de Granada (ACC, Caj. W, n. 132).

(4) Sobre el enfrentamiento entre los carniceros y el obispo y cabildo de la catedral de Córdoba, vid. PADILLA GONZÁLEZ, Jesús, El conflicto de las carnicerias de cristianos de Cordoba o el fracaso de una lucha antimonopolistica (1281-1311), en Axerquia, $\mathrm{n}^{\circ} 1$ (Córdoba, junio 1981), 121-155. La pugna entre el concejo y los dueños de las carnicerias es analizada por el mismo autor en Relaciones y pautas de comportamiento de las autoridades civiles $y$ eclesiásticas de la ciudad de Córdoba ante un conflicto de intereses: el monopolio de la comercialización de la carne (1311-1457), en Axerquia, $n^{\circ} 4$ (abril 1982), 143-182.

(5) QUINTANILLA RASO, M." Concepción, Notas sobre el comercio urbano en Córdoba durante la Baja Edad Media, en Actas I Congreso de Historia de Andalucia. Andalucia Medieval, I (Córdoba, 1978), 417.

(6) BCC, Ms. 125, ff. 15 r.-16 v.

(7) Ibid.

(8) No hemos hallado ningún documento en el Archivo Municipal de Sevilla que confirme esta donación, ni nada en este sentido se menciona en las Ordenanzas de Sevilla (hemos consultado para este particular las Ordenanzas de Sevilla, editadas en Sevilla, 1975, por la Oficina Técnica de Arquitectura e Ingenieria, S. A., ed. facsímil de las Ordenanças de Seuilla, impresas en esta ciudad en 1632, por Andrés Grande, ff. 139 r.-141 v.). 
(9) Sobre la concesión del monopolio de la comercialización de la alfareria y los problemas derivados de ésta, vid. PADILLA GONZALEZ, Jesús, El conflicto de las ollerias de Córdoba (1281-1304). Notas para un estudio socioeconómico de la Baja Edad Media cordobesa, en Axerquia, $\mathrm{n}^{\circ} 2$ (junio 1981), 301-307. Sobre la ola de anticlericalismo que se desató como consecuencia de la monopolización del comercio de la carne y el barro, vid. NIETO CUMPLIDO, Manuel, La Libertad religiosa en Córdoba (Córdoba, 1968), 10-12.

(10) ACC, Caj. W, n. 1.

(11) Los carniceros y concejo de la ciudad culparon de esta espectacular subida al sistema de arrendamiento, pero no debemos olvidar que estamos hablando de una época en la que se produjo una notable devaluación de la moneda y un profundo proceso inflacionista (cfr., entre otros, GRASSOTTI, Hilda, Miscelánea de estudios sobre instituciones castellano-leonesas (Bilbao, 1978). De esta obra destacamos los artículos titulados «Para la historia del botin y de las parias: la baja del oro en Europa", 133-221, especialmente el capítulo III, titulado "Crisis Castellana y zigzagueos granadinos", 177 y ss. $Y$ en segundo lugar el artículo "Sobre la moneda de Castilla en la época de Don Rodrigo Ximénez de Rada", 422-431. También en VICENS VIVES, J., Historia de España y de América, II (tomo titulado Baja Edad Media) (Barcelona, 1961), 94-106. Al mismo tiempo que se producia esta inflación se dio un aumento desmesurado del número de los carniceros, pues en 1281 eran 16 y en 1311 eran 33 , sin que se produjese un aumento paralelo del número de tablas de carnicerias (cfr. PADILLA GONZALLEZ, J., El conflicto de las carnicerias..., 151 y s.

(12) Ibid., 140 y ss.; BCC, Ms. 125, ff. 15 r.-16 v.; CMC, 1311, febrero 25, Burgos.

(13) ACC, Caj. W, n. 10. Por estar muy deteriorada no se conserva más que el año de su expedición, no el mes, ni el dia (CMC, 1331 (s.m.). (s.d.), (s.l.). Por otra segunda provisión real fechada el 20 de marzo del mismo año sabemos que algunos carniceros habian sido apresados, mientras otros se hallaban escondidos o habian huido de la ciudad temiendo las represalias de los oficiales del concejo porque se habian declarado en huelga y no habían abastecido convenientemente de carne a la ciudad (ACC, Caj. W, n. 11; CMC, 1331, marzo 20, Sevilla).

(14) ACC, Caj. T, n. 151. Este documento tiene perdida su fecha y no da el apellido del obispo. Sin embargo, por su tipología y por hallarse en un contexto documental correspondiente al siglo XIV, estimamos que es de esta centuria. En el "Trescientos" existen dos obispos de nombre "Juan": el primero, don Juan Pérez (1336-1346); el segundo, don Juan Fernández Pantoja (1379-1397) (vid. NIETO CUMPLIDO, M., La elección de obispos en Córdoba en la Baja Edad Media, en Andalucia Medieval: Nuevos estudios (Córdoba, 1979), 73-103; GÓMEZ BRAVO, Juan, Catálogo de los obispos de Córdoba (Córdoba, 1778); RUANO, Francisco, Episcopologio Cordubense, Biblioteca Colombina de Sevilla, ms. 83-6-29). Consideramos que el don Juan de nuestro arrendamiento es el primero, pues todos los documentos del Cajón T del Archivo de la catedral de Córdoba, lugar en el que se encuentra el arrendamiento que comentamos, están fechados en el pontificado del mencionado obispo y el más tardío no es de fecha posterior a 1450, por lo que el arrendamiento podernos datarlo entre 1336 y 1346.

(15) Cfr. NIETO CUMPLIDO, Manuel, La crisis demográfica y social del siglo XIV en Córdoba, en Anales del instituto Luis de Góngora, 3 (Córdoba, 1973), 31-32.

(16) ACC, Caj. W, n. 15; CMC, 1354, octubre 31, Córdoba.

(17) NIETO CUMPLIDO, Manuel, Op. c., 32.

(18) ACC, Caj. I, n. 457-1, f. 5 v;; CMC, 1396, febrero 5, Córdoba.

(19) Una acabada prueba de lo que decimos la tenemos documentada el 30 de enero de 1393 (ACC, Caj. l, n. 393, f. 35 v.; CMC, 1393, enero 30, Córdoba). En esta fecha el cabildo puso en aimoneda el arrendamiento de una tabla de carnicería: en el primer cabildo el chantre ofreció $210 \mathrm{mrs}$. y Gonzalo Gil, compañero, 230; en el segundo, no se superó la puja; pero en el tercero, el compañero Juan Rodríguez ofreció $\mathbf{4 5 0} \mathrm{mrs}$. Siete dias más tarde, la mencionada tabla fue arrendada a Yagüe Rodriguez por 500 mrs. (ACC, Caj. W, n. 16; CMC, 1393, febrero 6, Córdoba). Como podemos comprobar, hubo un margen de $50 \mathrm{mrs}$. de beneficio obtenido por el intermediario.

(20) Es muy frecuente observar el hecho de que la cantidad pujada en el remate es la misma que la del recaudo, lo que nos hace pensar que no siempre habia beneficio para el clérigo intermediario que realizaba la gestión. Probablemente existiría otro sistema de compensar los buenos oficios de éste no constatable en la documentación. Un modelo de ello nos lo ofrece el siguiente testimonio: el 16 de diciembre de 1392 el cabildo puso en almoneda una casilla de 
carnicería que vacó por muerte de la madre de Diego Rodríguez, carnicero, la cual se remató en Alfonso Ferrández, compañero, «para un lego", por $200 \mathrm{mrs}$. de renta anual (ACC, Caj. I, leg. IV, n. 395, f. 31 v.; CMC, 1392, diciembre, 16, Córdoba). El 15 de enero de 1393 hicieron recaudo de ella Ferrant Martinez y su esposa Estefania Alfonso, por la misma cantidad (ACC, Caj. l, leg. IV, n. 395 , f. 33 v.; CMC, 1393, enero 15, Cordoba).

(21) Se dio con frecuencia. Una muestra de ello lo tenemos el 3 de enero de 1392, fecha en la que el cabildo concedió al maestrescuela la autorización para que Juan Muñoz el Viejo pudiese renunciar a su tabla en favor de su hijo Alfón Muñoz, ordenando, asimismo, que al siguiente viernes el canónigo Pero Ruiz y el compañero Ruy Pérez tomase recaudo de ésta (ACC, Caj. I, leg. IV, n. 395, f. 2 r.; CMC, 1392, enero 3, Córdoba).

(22) Con fecha de 11 de marzo de 1392 encontramos un ejemplo similar al indicado en la nota anterior, pero en él se ofrecen dos particularidades: la primera es que una vez aceptada la renuncia se nombra una comisión para ir a recibirla personalmente y por escrito del carnicero saliente; la segunda es el aumento de la renta en $200 \mathrm{mrs}$. (ACC, Caj. I, leg. IV, n. 395, f. 10 r.; CMC, 1392, marzo 11, Córdoba), quedando el nuevo arrendamiento en $1.400 \mathrm{mrs}$. de renta anual (ACC, Caj. W, n. 14; CMC, ibid.).

(23) BCC, Ms. 166, f. 63 rv.; CMC, 1396, agosto 2, Córdoba.

(24) Así tenemos el caso de Pedro Fernández, que tenia arrendada la primera tabla a mano derecha de la carnicería de San Salvador desde 1354 (ACC, Caj. W, n. 15; CMC, 1354, octubre 31, Córdoba), y en 1364 arrendó la segunda de la izquierda de la misma carnicería (ACC, Caj. W. n. 18; $C M C, 1364$, diciembre 20, Córdoba). Otro caso similar se dio en 1476, fecha en la que Gonzalo García arrendó de por vida tres tablas en la carnicería de Santa María (ACC, Caj. W, n. 70; CMC, 1476, octubre 2, Córdoba).

(26) ACC, Caj. W, n. 32; CMC, 1438, junio 23, Córdoba.

(26) ACC, Caj. W, n. 17; CMC, 1400, septiembre 22, Córdoba. Citamos este documento por pruritos de concreción, pero son cláusulas que se repiten mecánicamente en todos los contratos de arrendamientos. Sobre los documentos de las Carnicerias de Córdoba, vid. PADILLA GONZALEZ, Jesús, Catálogo de los documentos del cajón $W$ del Archivo de la Catedral de Córdoba, original mecanografiado en ACC. 